\title{
Multiscale Simulation for Visible Light Communication using Perovskite Metasurface
}

\author{
Raphaël Mermet-Lyaudoz \\ Univ Lyon, École Centrale de Lyon \\ CNRS, INSA Lyon, INL, UMR 5270 \\ 69130 Ecully, France \\ raphael.mermet-lyaudoz@ec-lyon.fr
}

Anne Julien-Vergonjanne

XLIM Laboratory, UMR CNRS 7252

University of Limoges

87060 Limoges cedex

anne.julien-vergonjanne@unilim.fr

Stéphanie Sahuguède

XLIM Laboratory, UMR CNRS 7252

University of Limoges

87060 Limoges cedex

stephanie.sahuguede@unilim.fr

\author{
Pierre Combeau \\ XLIM Laboratory, UMR CNRS 7252 \\ University of Poitiers \\ 86073 Poitiers, France \\ pierre.combeau@univ-poitiers.fr \\ Christian Seassal \\ Univ Lyon, École Centrale de Lyon \\ CNRS, INSA Lyon, INL, UMR 5270 \\ 69130 Ecully, France \\ christian.seassal@ec-lyon.fr
}

\author{
Emmanuel Drouard \\ Univ Lyon, École Centrale de Lyon \\ CNRS, INSA Lyon, INL, UMR 5270 \\ 69130 Ecully, France \\ emmanuel.drouard@ec-lyon.fr \\ Hai Son Nguyen \\ Univ Lyon, École Centrale de Lyon \\ CNRS, INSA Lyon, INL, UMR 5270 \\ 69130 Ecully, France \\ hai-son.nguyen@ec-lyon.fr
}

\begin{abstract}
This article presents an original multi-scale simulation approach, enabling to rigorously compute the far-field radiation from emerging halide perovskite light-emitting diodes integrating a metasurface active layer, and subsequently to evaluate its impact on the propagation channel characteristics at the scale of an indoor environment, both in terms of coverage and time dispersion. Our results show that the high versatility of the metasurface design parameters allows the outage probability to be divided up to a factor 2 without degrading the temporal dispersion of the optical channel in visible light communication scenarios.
\end{abstract}

Index Terms-Halide perovskite, nanophotonics, metasurface, multi-scale simulation, visible light communications, MonteCarlo Ray-Tracing.

\section{INTRODUCTION}

About 10 years ago [1], the solid-state lighting revolution for indoor illumination has promoted the emergence of visible light communications (VLC) or related light fidelity ( $\mathrm{LiFi}$ ), where light-emitting diode (LED) can provide both illumination and communication. Communication performance of theses systems are significantly impacted by the optical characteristics of the source, in both terms of lighting levels and radiation pattern [2].

Recently, the use of organic light emitting diodes (OLEDs) have been proposed mainly due to their integration flexibility and large active areas [3], [4]. However, their modulation bandwidth is low, limiting their use for high data rates applications.

This work was funded by the french National Research Agency under the grant agreement no ANR-18-CE24-0016.
Halide perovskites, as emerging materials with excellent optical and electrical properties, are prominent candidates for active medium in optoelectronic devices. The popularity of halide perovskites is often symbolized by its rising in photovoltaic applications as an alternative to silicon [5], with the power conversion efficiency of perovskite based solar cells now exceeds $25 \%$. On the other hand, as direct-bandgap semiconductors, they are now also considered as an alternative to III-V semiconductor for emitting devices such as microlasers [6] and LED [7]. The record of halide perovskite based LED external efficiency has recently exceeded the $20 \%$ milestone [8]. Indeed, these materials combine strengths of the inorganic semiconductors and organic semiconductors, likely to solve the contradiction that high charge carrier mobility and large stimulated emissions are required for lasing devices but are not found generally in the same material. In particular, unlike OLEDs, halide perovskites LEDs exhibit key properties like long carrier diffusion lengths [9] and high refractive index, which is an important prerequisite to control lightmatter interaction. Moreover, unlike inorganic materials like GaN-based heterostructure, halide perovskite layers can be directly patterned at the sub-wavelength scale using scalable and high throughput technological processes like nanoimprint lithography [10]. The integration of such patterns in LEDs enables to control both the light emission spectra of halide perovskite thin films and their far-field emission pattern [11].

To evaluate communication performance, these light emission properties are first predicted using electromagnetic simulations. These characteristics are then used as input data in simulation tools of light propagation to assess the propagation 

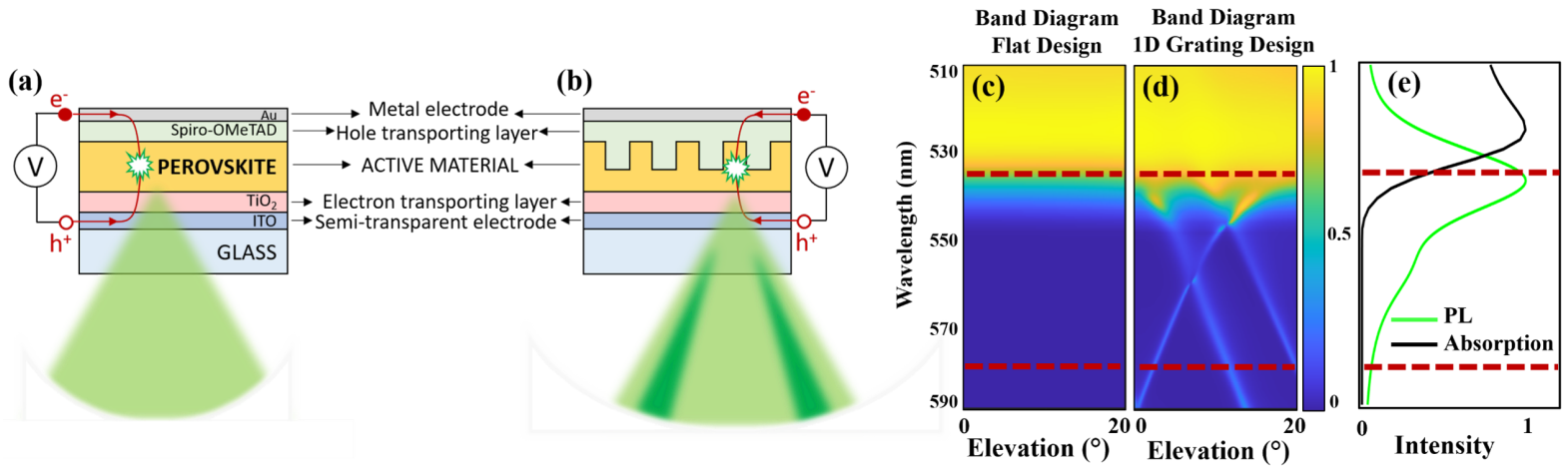

Fig. 1. Schemes of the (a) flat and (b) structured metasurface halide perovskite LED. Band diagram of the absorption within a (c) flat and (d) 1D grating structured perovskite layer. (e): Spectra of the absorption and the photoluminescence of the $\mathrm{CH}_{3} \mathrm{NH}_{3} \mathrm{PbBr}_{3} \mathrm{perovskite}$

channel in realistic environments. The main tools are based on Monte-Carlo ray-tracing (MCRT) algorithms which, from a 3D geometrical model of the propagation environment and the optical properties of the materials, allow computing the line-of-sight (LOS) and multi-reflected contributions between the source and the receiver [12].

This work presents first the use of resonant metasurfaces to engineer the photoluminescence (PL) emission of halide perovskite thin films. These metasurfaces are sub-wavelength periodic lattice which are directly obtained by patterning the halide perovskite thin film by thermal nanoimprint lithography. Their use allows enhancing the PL of perovskite and controlling on-demand the far-field emission pattern from quasi-isotropic emission to highly directional one. Then, these optical source data are considered in MCRT tool [13]-[15] to characterize the propagation channel in terms of optical DC gain and root mean square delay spread (DRMS). Thus, we propose a multi-scale simulation approach, from the device to the far-field propagation, with the perspective of efficient conception of halide perovskite sources in order to match specific VLC applications.

The rest of the article is organized as follows. Section II presents the conception process of the metasurface halide perovskite LED. Section III describes the channel modelling approach by presenting first the integration of metasurface halide perovskite LED characteristics in the MCRT tool in Section III-A, then the considered propagation environment in Section III-B, and finally by discussing the obtained results in Section III-C. Conclusions and perspectives are given in Section IV.

\section{Metasurface Halide Perovskite LED}

We first introduce the material stack in a standard halide perovskite LED. As depicted in Fig. 1(a,b), the LED stack consists of: $\mathrm{Au}$ and Indium Tin Oxyde (ITO) as contacts, $\mathrm{TiO}_{2}$ and Spiro-OMeTAS as transporting layer for respectively electrons and holes, and halide perovskite as the active material where the carrier recombination leading to light emission takes place. As a proof-of-concept, the chosen perovskite is $\mathrm{CH}_{3} \mathrm{NH}_{3} \mathrm{PbBr}_{3}$ which is one of the most popular and stable perovskites with strong emission in the green wavelength range. Emitted light is collected from the ITO side. Thicknesses of each material are kept constant for the different designs $\left(\mathrm{h}_{A u}=200 \mathrm{~nm}, \mathrm{~h}_{\text {Spiro }}=150 \mathrm{~nm}, \mathrm{~h}_{\text {Perovskite }}=270\right.$ $\mathrm{nm}, \mathrm{h}_{\mathrm{TiO}_{2}}=20 \mathrm{~nm}, \mathrm{~h}_{I T O}=200 \mathrm{~nm}$ ) - rather flat or having perovskite layer patterned into metasurface structure.

The rigorous coupled wave analysis (RCWA) [16] suits well for the simulation of the stacks periodically patterned under plane wave illumination. We have used the $S^{4}$ code [17] available in the Solcore package [18] to compute the absorption within the perovskite layer. According to Kirchhoff's law of thermal radiation, the emission in the $(\theta, \phi)$ space with $\theta \in$ $[0,360]$ the azimuth and $\phi \in[0,90]$ the elevation equals the absorption in the same space. Such a result is still commonly used for out-of-equilibrium active devices such as LED. Then absorption $A(\theta, \phi, \lambda)$ is obtained using RCWA by applying the Poynting's theorem to the perovskite material area. Far-field of the emitted light is then obtained integrating the product of the absorption and the spectrum of the PL of the perovskite we measured, along the wavelength range of interest $\left[\lambda_{1}, \lambda_{2}\right]=[535$ $\mathrm{nm}, 580 \mathrm{~nm}$ ] (cf. area delimited by dashed red lines in Fig. 1(ce)), as:

$$
\operatorname{Farfield}(\theta, \phi)=\int_{\lambda_{1}}^{\lambda_{2}} A(\theta, \phi, \lambda) P L(\lambda) \mathrm{d} \lambda
$$

The metasurface designs investigated in this work, in addition to the flat slab, are sub-wavelength periodic lattices of 1D grating, 2D square, 2D triangular and 2D honeycomb whose schemes are depicted in Fig. 2(a-e). The introduction of a periodic pattern in the active material triggers optical resonances to occur enhancing photons absorption and emission phenomena for given $\theta$ and $\phi$. For patterned LED device, the emitted photons can be coupled to Bloch resonances of the periodic metasurface. Such a coupling has two-fold effects: i) Enhance the extraction efficiency [19] ii) Shape the far-field pattern following the photonic bandstructure. Although an improvement of light extraction with metasurface design is hinted (see Fig. 2(f-j)), here we only focus on the engineering of far-field pattern for VLC applications. The tuning of the geometrical parameters of the pattern is a way to engineer the shape of the 
Flat

(a)

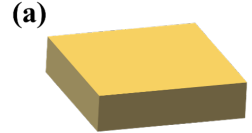

(f)

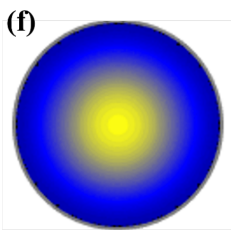

1D Grating

(b)

(b)
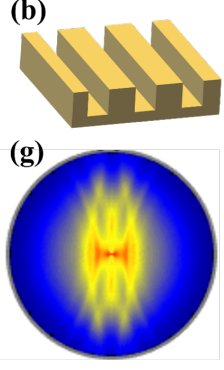

2D Square

(c)
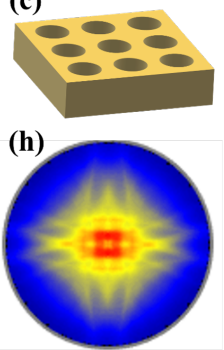

2D Triangular

(d)
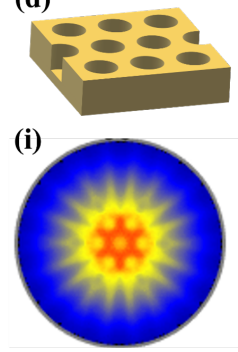

2D Honeycomb

(e)

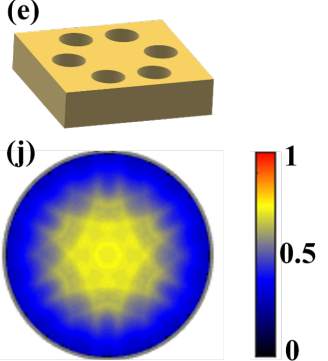

Fig. 2. (a), (b), (c), (d) and (e): Schemes of the different patterns within the perovskite layer. Depths of all patterns is $135 \mathrm{~nm}$. 1D grating exhibits a period of $295 \mathrm{~nm}$ and a filling factor of $0.5 .2 \mathrm{D}$ square $($ period $=300 \mathrm{~nm}$, radius $=90 \mathrm{~nm}$ ). 2D triangular $($ period $=310 \mathrm{~nm}$, radius $=90 \mathrm{~nm}) .2 \mathrm{D}$ honeycomb (period $=460 \mathrm{~nm}$, radius $=80 \mathrm{~nm})$. $(\mathrm{f}),(\mathrm{g})$, (h), (i) and $(\mathrm{j})$ : Far-field emission of the corresponding geometries.

(a)

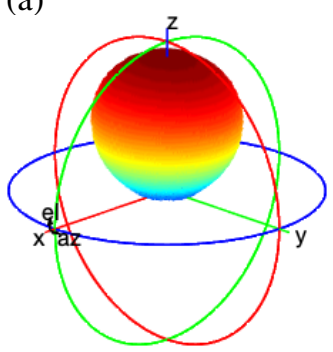

(b)

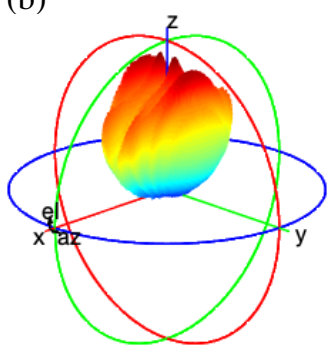

(c)

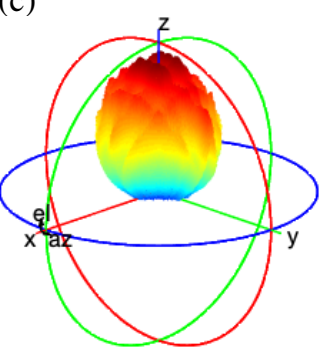

(d)

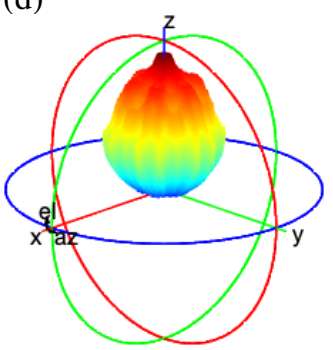

(e)

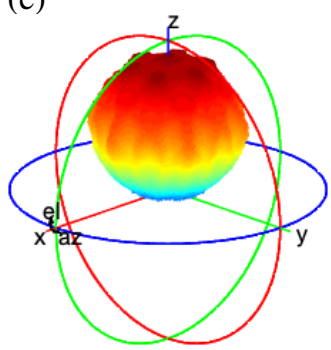

Fig. 3. Radiation patterns of the different LED structure: (a) flat, (b) 1D grating, (c) 2D square, (d) 2D triangular, and (e) 2D honeycomb.

LEDs beam. For instance, dealing with symmetries, the shapes of the far-field in Fig. 2(f-j) exhibit the same symmetries as the patterns in Fig. 2(a-e). Moreover, the photonic bandstructure can be engineered on-demand within the spectral region of interest to exhibit various characteristics such as slow-light and linear dispersion [20], or even peculiar ones such as flatband, Dirac cones and multivalley characteristics [21], [22].

\section{ChanNEL MODELLING}

\section{A. Propagation simulation}

To evaluate the impact of those metasurface halide perovskite LEDs, we simulate the channel impulse response (CIR) $h(t)$ for a given link between a couple of emitter and receiver. We adopt a modelling approach based on a stochastic Monte-Carlo method, associated with ray-tracing algorithm, to numerically determine, from analytical models of reflection (bidirectional reflectance distribution function (BRDF)) and propagation, the propagation delay and the received power for LOS and non-LOS (NLOS) paths, i.e. reflected paths over all the elements inside the propagation environment. In this paper, we consider the MC ray-shooting [23] simulation algorithm, with $10 \mathrm{e} 6$ launched rays, a maximum reflection depth of 3 and a time resolution of $0.2 \mathrm{~ns}$.

Thus, the simulation process consists first in integrating in the light propagation simulator the simulated radiation patterns of the different developed LED structures presented in Fig. 3, which have been computed based on the process presented in Section II. Corresponding to the flat slab structure, Fig. 3(a) exhibits a pattern very close to the standard Lambertian one.
It can be viewed as a reference case. These radiation patterns are computed for a set of incident directions, deterministically determined with a constant angular step, in both elevation and azimut. Since the MCRT algorithm leads to consider random incident directions from the optical source, in which the radiated luminance is usually not known, it is simply interpolated from the known values in neighboring directions. Finally, The radiation patterns are obviously normalized by the total radiated power computed by $\mathrm{MC}$ integration over the hemisphere covering the LED.

In [24], a recent study highlighted the impact of the area of the transmitter's excitation surface onto the channel DC gain, which becomes significant with its increase. Its shape, circular or rectangular, can also be of importance. These two parameters are taken into account in our MCRT simulations. Next lines present the corresponding process in case of only LOS link.

$G_{T_{x}}(\vec{v})$ being the normalized radiation pattern of the optical source $T_{x}$, defining so the relative radiated power in direction $\vec{v}$, the overall received power $P_{R_{x}}$ by the receiver $R_{x}$ is given by:

$$
P_{R_{x}}=P_{t} \iint_{A_{T_{x}}, A_{R_{x}}} G_{T_{x}}(\overrightarrow{x y}) V(x, y) \frac{\left|\overrightarrow{x y} \cdot \overrightarrow{n_{r}}\right|}{\|\overrightarrow{x y}\|^{3}} d \mu(x) d \mu(y),
$$

where $P_{t}$ is the total emitted optical power, $A_{T_{x}}$ and $A_{R_{x}}$ are the areas of the emitter/receiver, $x$ and $y$ two given points onto the emitter/receiver' surfaces, $\overrightarrow{x y}$ the vector from $x$ to $y$, $\overrightarrow{n_{r}}$ the normal at the receiver' surface at $y, d \mu(x)$ and $d \mu(y)$ 
two elementary areas around $x$ and $y$, and $V$ the visibility function defined as:

$$
V(x, y)=\left\{\begin{array}{ll}
1 & \text { if } x \text { and } y \text { are visible from each other } \\
0 & \text { else }
\end{array} .\right.
$$

The conventional MC estimator $\widehat{P}_{R_{x}}$ of $P_{R_{x}}$ computed for $N$ samples is:

$$
\widehat{P}_{R_{x}}=\frac{P_{t}}{N} \sum_{i=1}^{N} \frac{G_{T_{x}}\left(\overrightarrow{x_{i} y_{i}}\right) V\left(x_{i}, y_{i}\right) \frac{\left|\overrightarrow{x_{i} y_{i}} \cdot \overrightarrow{n_{r}}\right|}{\mid \overrightarrow{x_{i} y_{i}} \|^{3}}}{p_{x}\left(x_{i}\right) p_{y}\left(y_{i}\right)}
$$

where $x_{i}$ and $y_{i}$ are two random points onto the $T_{x}$ and $R_{x}$, surfaces, computed from the considered probability density functions (PDF) $p_{x}$ and $p_{y}$ respectively. The genericity of MC integration method leads to the fact that the MC estimator given in (4) is valid for any PDF. Although the estimator's variance is impacted by the choice of a specific PDF and as we have no a priori information on $G_{T_{x}}(\vec{v})$, we have adopted conventional uniform PDF, i.e.:

$$
p_{x}\left(x_{i}\right)=\frac{1}{A_{T_{x}}}, \quad p_{y}\left(y_{i}\right)=\frac{1}{A_{R_{x}}} .
$$

Finally, the algorithms to uniformly sample a point onto a rectangular or circular surface are obvious. There expressions can be found in [25].

\section{B. Propagation environment}

The simulation environment, illustrated in Fig. 4, is the Barry-A scene first proposed in [26]. It consists of a paralepipedic room of $5 \times 5 \times 3 \mathrm{~m}$. The optical source is at the ceiling center and points towards the ground. The receivers are photodiodes with a $1 \mathrm{~cm}^{2}$ sensitive area and a $60^{\circ}$ field of view (FoV), defined as the half-detection power semi-angle. We consider a set of receiving positions on the ground, located on a discrete grid with a fixed $20 \mathrm{~cm}$ step, corresponding to a set of 625 reception positions. All the geometrical and optical parameters of the environment are detailed in Table I.

\section{Results}

The CIRs have been simulated between the emitter and the set of receiving positions presented in Fig. 4, according to each of the five LED structures presented in Section II, allowing to draw in Fig. 5 maps of channel DC gain $H_{0}$ defined as:

$$
H_{0}=\int_{0}^{\infty} h(t) \mathrm{d} t .
$$

Based on the central limit theorem, $H_{0}$ was found in the confidence interval $\left[0.99 \widehat{H_{0}}, 1.01 \widehat{H_{0}}\right]$ with a probability of $95 \%, \widehat{H_{0}}$ being the $\mathrm{MC}$ estimator of $H_{0}$. Theses maps have a similar behavior according to the different LEDs, as indicated as example for flat and 2D honeycomb structures in Fig. 5(a) and (c) respectively, which present very close gain distributions inside the room. This is confirmed by the PDFs of the channel DC gain presented in Fig. 6 (plain line curves). This figure highlights a minimum gain of about $-59 \mathrm{~dB}$.

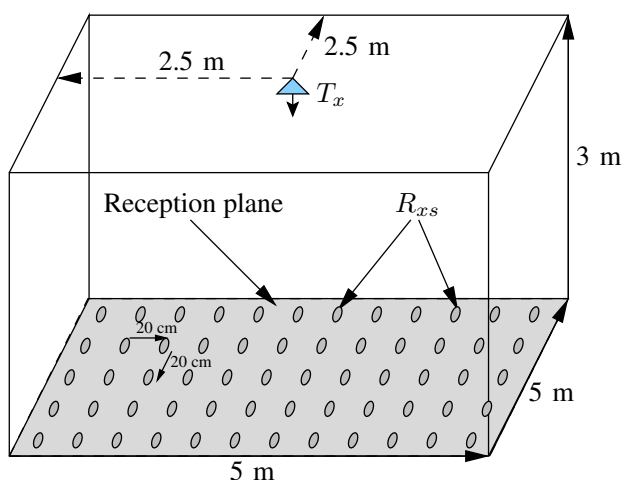

Fig. 4. Simulation environment.

TABLE I

PARAMETERS OF THE PROPAGATION ENVIRONMENT [?].

\begin{tabular}{ll}
\hline Parameters & values \\
\hline \hline Room size & $5 \times 5 \times 3 \mathrm{~m}$ \\
BRDF of all surfaces & Lambertian, i.e. purely diffuse \\
Reflectance of walls and ceiling & 0.8 \\
Reflectance of floor & 0.3 \\
$T_{x}$ coordinates & $(2.5,2.5,2.99)$ \\
$T_{x}$ power & $1 \mathrm{~W}($ normalized $)$ \\
$T_{x}$ geometrical shape / area & Rectangular / $1 \mathrm{~cm}^{2}$ \\
Number of receiving positions & $625(25 \times 25)$ \\
Spacing of neighboring receiver & $20 \mathrm{~cm}$ \\
positions & \\
$R_{x}$ geometrical shape / area & Circular / $1 \mathrm{~cm}^{2}$ \\
FoV of the receivers & $60^{\circ}$ \\
\hline
\end{tabular}

Even though it cannot be guaranteed in a real environment, due to mobility for instance, the best communication performance level is obviously obtained when the LOS link exists. Fig. 6 also illustrates the PDFs of the LOS contribution to the channel DC gain (dashed line curves). It appears that the LOS gain can be lower that the minimum channel gain $(-59 \mathrm{~dB})$, and in this case it is compensated by the reflections over the room surfaces. Also, the maximum occurrence of gain value is still about $-59 \mathrm{~dB}$ (dotted line).

If one consider this value as a target limit to guarantee the best level of performance, even in case of LOS blockage, the cumulative density function (CDF) of the multi-reflected contributions according to the LED structures, presented in Fig. 7, allows determining the outage probability $P_{\text {out }}$, i.e. the probability to have a channel gain value less than this targeted one. Theses curves clearly indicate significant gap between the $P_{\text {out }}$ values, which are summarized in Table II. The 2D triangular and flat LED structures give the highest $P_{\text {out }}$ values of 82.24 and $79.84 \%$ respectively. The 1D structure is a little better with $71.52 \%$, whereas the 2D square and honeycomb structures are the most interesting, with a significantly weaker $P_{\text {out }}$ values of 62.72 and $44.16 \%$ respectively. Thus, the best structure, i.e $2 \mathrm{D}$ honeycomb, allows to divide the $P_{\text {out }}$ by a factor of about 2 with regards to flat one. 
(a)

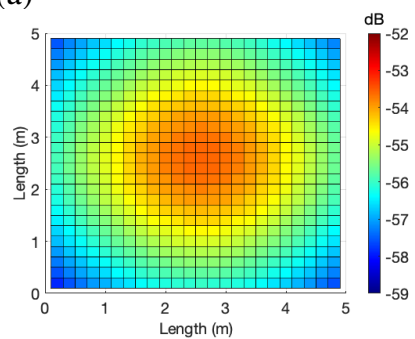

(b)

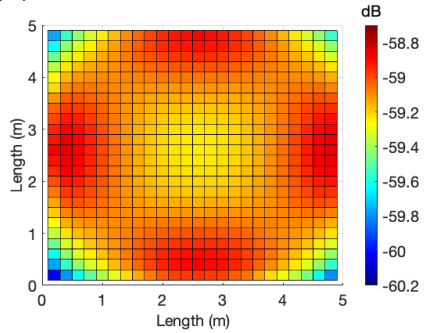

(c)

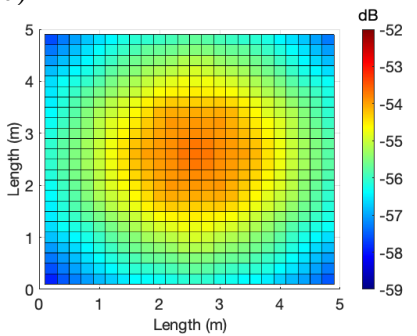

(d)

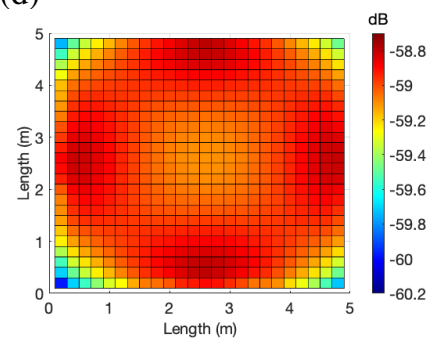

Fig. 5. Maps of Channel DC gain according to: (a) only LOS contribution and Flat structure, (b) only multi-reflected contributions and Flat structure, (c) only LOS contributions and 2D honeycomb structure, and (d) only multi-reflected contributions and 2D honeycomb structure.

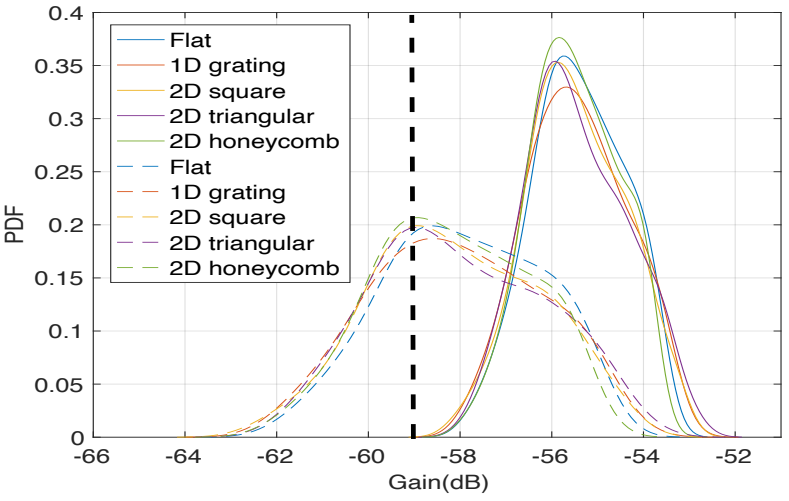

Fig. 6. PDFs of the channel DC gain (plain lines) and the LOS contribution (dashed lines) to the channel DC gain according the LED structures.

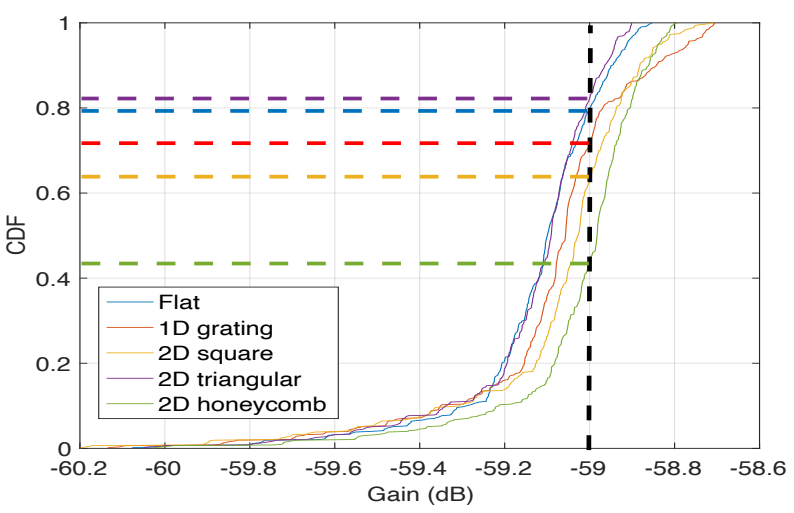

Fig. 7. CDFs of the only multi-reflected contributions to the channel DC gain according the LED structures.

This interesting result is obtained thanks to the reflected contributions which are the highest for the $2 \mathrm{D}$ honeycomb structure, as Fig. 5(b) and (d) illustrate it for flat and 2D honeycomb structures respectively.

As last part of this study, we now present the impact of LEDs' structure onto the time dispersive character of the channel, by computing its DRMS [12] expressed for recall by:

$$
D R M S=\sqrt{\frac{\int(t-\mu)^{2} h^{2}(t) d t}{\int h^{2}(t) d t}}, \mu=\frac{\int t h^{2}(t) d t}{\int h^{2}(t) d t},
$$

TABLE II

OUTAGE PROBABILITY FOR A NLOS TARGET GAIN OF -59 DB ACCORDING TO THE LED STRUCTURES.

\begin{tabular}{lc}
\hline LED structures & $\boldsymbol{P}_{\text {out }}: \mathbf{P}\left(\boldsymbol{H}_{\mathbf{0}}<-\mathbf{5 9} \mathbf{d B}\right)$ \\
\hline \hline Flat & $79.84 \%$ \\
1D grating & $71.52 \%$ \\
2D square & $62.72 \%$ \\
2D triangular & $82.24 \%$ \\
2D honeycomb & $44.16 \%$ \\
\hline
\end{tabular}

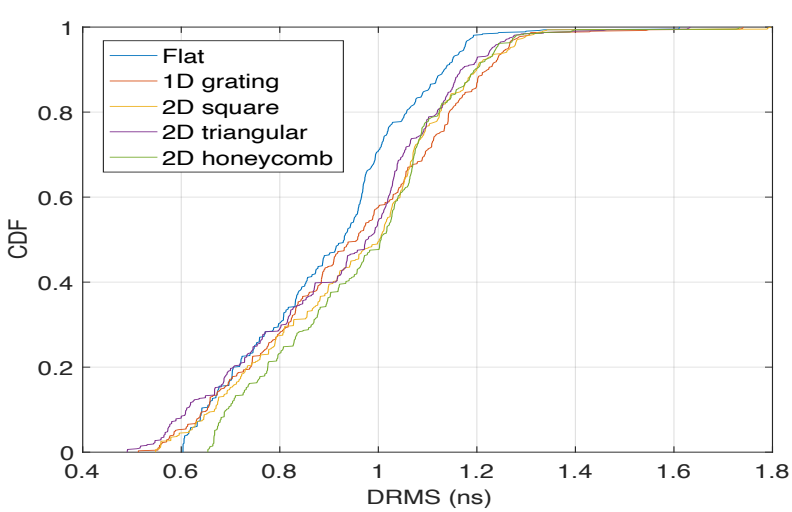

Fig. 8. CDFs of the DRMS according the LED structures.

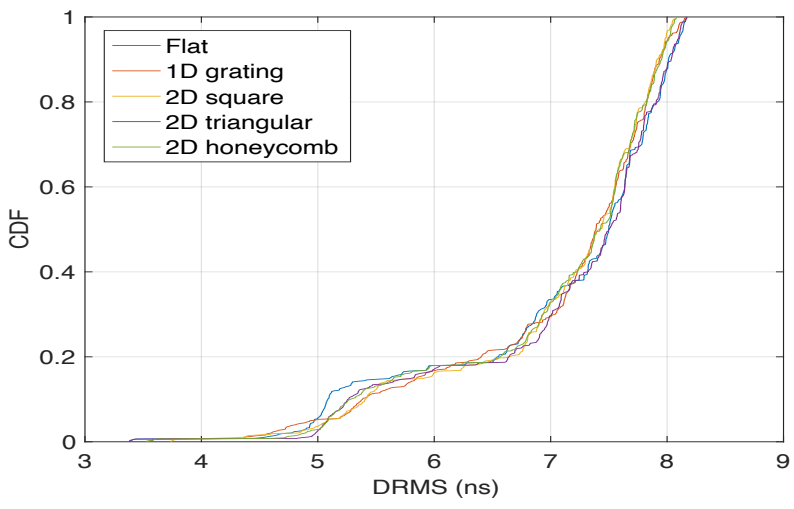

Fig. 9. CDFs of only multi-reflected contributions to the DRMS according the LED structures.

with $\mu$ the mean excess delay. 
Fig. 8 shows the CDF of the DRMS according to the LED structures. It appears that the structure of the LEDs has very little impact on the DRMS with a maximum difference of only $0.2 \mathrm{~ns}$ which corresponds to $0.1 \%$ of the maximum DRMS value. Even if this first result is interesting, since it shows that the LED structuring does not degrade the channel performance in terms of temporal dispersion, it is obtained in the favorable configuration where the LOS link is always existing.

Because reflected contributions have more similar power levels between each other, NLOS configurations are more conducive to high levels of DRMS. To check the impact of the LED structuration in the NLOS case, Fig. 9 shows the CDF of the only multi-reflected contributions. As expected, the levels of DRMS are significantly higher than in LOS case, but the difference between the different LED structures are still weak, with a maximum difference of about $0.4 \mathrm{~ns}$, being only $0.05 \%$ of the maximum DRMS value.

\section{CONCLUSION}

By implementing a modeling approach, we investigated the impact of metasurface halide perovskite LED design on the characteristics of the optical wireless propagation channel. The optical properties of the designed components were included in an optical propagation simulator based on the MCRT method to determine the characteristics of the transmission channel. Overall, we have underlined that the structuring of the LED, considering normalized radiation patterns, allows optimizing the coverage by dividing the outage probability up to a factor 2 , without degrading the temporal dispersion of the channel. These first results illustrate the interest of such a multi-scale approach that we will deepen by studying the impact of other parameters related to the design of LEDs. Beyond its influence on the emission pattern, we will for instance investigate the impact of perovskite metasurface geometrical parameters on the emitted power and the electrical consumption, and take it into account in propagation simulation to evaluate its impact on communication performance in more complex optical transmission scenarios.

\section{REFERENCES}

[1] M. Z. Afgani, H. Haas, H. Elgala, and D. Knipp. Visible light communication using OFDM. In $2^{\text {nd }}$ International Conference on Testbeds and Research Infrastructures for the Development of Networks and Communities, 2006. TRIDENTCOM 2006., pages 6 pp.-134, 2006.

[2] D. Jupeng, I. Chih-Lin, and X. Zhengyuan. Indoor Optical Wireless Channel Characteristics With Distinct Source Radiation Patterns. IEEE Photonics Journal, 8(1):1-15, 2016.

[3] C. Hanjie and X. Zhengyuan. OLED Panel Radiation Pattern and Its Impact on VLC Channel Characteristics. IEEE Photonics Journal, 10(2):1-10, 2018.

[4] Z. N. Chaleshtori, S. Zvanovec, Z. Ghassemlooy, H. B. Eldeeb, and M. Uysal. A Flexible OLED VLC System for an Office Environment. In 2020 12th International Symposium on Communication Systems, Networks and Digital Signal Processing (CSNDSP), pages 1-5, 2020.

[5] J. Y. Kim, J-W. Lee, H. S. Jung, H. Shin, and N-G. Park. High-Efficiency Perovskite Solar Cells. Chemical Reviews, 120(15):7867-7918, 2020. PMID: 32786671

[6] Q. Wei, X. Li, C. Liang, Z. Zhang, J. Guo, G. Hong, G. Xing, and W. Huang. Recent Progress in Metal Halide Perovskite Micro- and Nanolasers. Advanced Optical Materials, 7(17):1900080, 2019.
[7] Q. Zhang, D. Zhang, Y. Fu, S. Poddar, L. Shu, X. Mo, and Z. Fan. Light Out-Coupling Management in Perovskite LEDs-What Can We Learn from the Past? Advanced Functional Materials, 30(38):2002570, 2020.

[8] Lin K., Xing J., and Quan L. N. et al. Perovskite light-emitting diodes with external quantum efficiency exceeding 20 per cent. nature, oct 2018.

[9] Y. Chen, H. T. Yi, X. Wu, R. Haroldson, Y. N. Gartstein, Y. I. Rodionov, K. S. Tikhonov, A. Zakhidov, X. Y. Zhu, and V. Podzorov. Extended carrier lifetimes and diffusion in hybrid perovskites revealed by Hall effect and photoconductivity measurements. Nature Communications, 7(1): $1-9$, aug 2016.

[10] A. Gharajeh, R. Haroldson, Z. Li, J. Moon, B. Balachandran, W. Hu, A. Zakhidov, and Q. Gu. Continuous-wave operation in directly patterned perovskite distributed feedback light source at room temperature. Opt. Lett., 43(3):611-614, Feb 2018.

[11] A. David, H. Benisty, and C. Weisbuch. Optimization of light-diffracting photonic-crystals for high extraction efficiency LEDs. IEEE/OSA Journal of Display Technology, 3(2):133-148, jun 2007.

[12] F. Miramirkhani and M. Uysal. Channel Modeling and Characterization for Visible Light Communications. IEEE Photonics Journal, 7(6):1-16, 2015.

[13] A. Behlouli, P. Combeau, and L. Aveneau. MCMC Methods for Realistic Indoor Wireless Optical Channels Simulation. IEEE/OSA Journal of Lightwave Technology, 35(9):1575-1587, May 2017.

[14] P. Combeau, S. Joumessi-Demeffo, A. Julien-Vergonjanne, L. Aveneau, S. Sahuguede, H. Boeglen, and D. Sauveron. Optical Wireless Channel Simulation for Communications Inside Aircraft Cockpits. Journal of Lightwave Technology, 38(20):5635-5648, oct 2020.

[15] S. Joumessi-Demeffo, S. Sahuguede, A. Julien-Vergonjanne, and P. Combeau. Performance Trade-offs of an Optical Wireless Communication Network Deployed in an Aircraft Cockpit. IEEE Open Journal of the Communications Society, 1:849-862, June 2020.

[16] M. G. Moharam and T. K. Gaylord. Rigorous coupled-wave analysis of planar-grating diffraction. JOSA, 71(7):811-818, 1981.

[17] V. Liu and S. Fan. S4 : A free electromagnetic solver for layered periodic structures. Computer Physics Communications, 183(10):22332244, 2012.

[18] D. Alonso-Álvarez, T. Wilson, P. Pearce, M. Führer, D. Farrell, and N. Ekins-Daukes. Solcore: a multi-scale, Python-based library for modelling solar cells and semiconductor materials. Journal of Computational Electronics, 17(3):1099-1123, 2018.

[19] S. Kar, N. Fadilah Jamaludin, N. Yantara, S. G. Mhaisalkar, and W. L. Leong. Recent advancements and perspectives on light management and high performance in perovskite light-emitting diodes. Nanophotonics, 2021.

[20] N-V. Hoang, A. Pereira, H. S. Nguyen, E. Drouard, B. Moine, T. Deschamps, R. Orobtchouk, A. Pillonnet, and C. Seassal. Giant Enhancement of Luminescence Down-Shifting by a Doubly Resonant RareEarth-Doped Photonic Metastructure. ACS Photonics, 4(7):1705-1712, jul 2017.

[21] H. S. Nguyen, F. Dubois, T. Deschamps, S. Cueff, A. Pardon, J. L. Leclercq, C. Seassal, X. Letartre, and P. Viktorovitch. Symmetry Breaking in Photonic Crystals: On-Demand Dispersion from Flatband to Dirac Cones. Physical Review Letters, 120(6), 2018.

[22] S. Cueff, F. Dubois, M. S. R. Huang, D. Li, R. Zia, X. Letartre, P. Viktorovitch, and H. S. Nguyen. Tailoring the Local Density of Optical States and Directionality of Light Emission by Symmetry Breaking. IEEE Journal of Selected Topics in Quantum Electronics, 25(3):1-7, 2019.

[23] A. Behlouli, P. Combeau, L. Aveneau, S. Sahuguede, and A. JulienVergonjanne. Efficient Simulation of Optical Wireless Channel Application to WBANs with MISO Link. Procedia Computer Science, 40(0):190-197, September 2014. Fourth International Conference on Selected Topics in Mobile and Wireless Networking (MoWNet2014).

[24] J. C. Valencia-Estrada, B. Béchadergue, and J. García-Márquez. Full Field Radiant Flux Distribution of Multiple Tilted Flat Lambertian Light Sources. IEEE Open Journal of the Communications Society, 1:927-942, 2020.

[25] M. Pharr, W. Jakob, and G. Humphreys. Physically Based Rendering: From Theory to Implementation. Morgan Kaufmann, 2016.

[26] J. R. Barry, J. M. Kahn, W. J. Krause, E. A. Lee, and D. G. Messerschmitt. Simulation of multipath impulse response for indoor wireless optical channels. IEEE Journal on Selected Areas in Communications, 11(3):367-379, apr 1993. 